\title{
Covariance structure of parabolic stochastic partial differential equations
}

\author{
Annika Lang • Stig Larsson · Christoph Schwab
}

Received: 11 October 2012 / Accepted: 12 May 2013 / Published online: 29 May 2013

(C) Springer Science+Business Media New York 2013

\begin{abstract}
In this paper parabolic random partial differential equations and parabolic stochastic partial differential equations driven by a Wiener process are considered. A deterministic, tensorized evolution equation for the second moment and the covariance of the solutions of the parabolic stochastic partial differential equations is derived. Well-posedness of a space-time weak variational formulation of this tensorized equation is established.
\end{abstract}

Keywords Stochastic partial differential equations - Random partial differential equations · Parabolic partial differential equations - Tensorized partial differential equations · Weak variational solutions

Mathematics Subject Classification (2010) $\quad 60 \mathrm{H} 15 \cdot 35 \mathrm{R} 60 \cdot 35 \mathrm{~K} 70$

\section{Introduction}

With general results on existence and uniqueness of solutions of stochastic partial differential equations being available (see, e.g., [5] and the references there), the

\footnotetext{
A. Lang $(\varangle)$. C. Schwab

Seminar für Angewandte Mathematik, ETH Zürich, Rämistrasse 101,

CH-8092 Zürich, Switzerland

e-mail: annika.lang@math.ethz.ch

C. Schwab

e-mail: schwab@math.ethz.ch

S. Larsson

Department of Mathematical Sciences, Chalmers University of Technology and

University of Gothenburg, SE-412 96 Gothenburg, Sweden

e-mail: stig@chalmers.se
} 
numerical solution of stochastic partial differential equations has received increasing attention in recent years. The most widely used numerical solution approaches are based on combinations of time stepping, space discretization, and sample path simulation. If the parabolic stochastic partial differential equation is linear and driven by $Q$-Wiener noise, the linearity of the stochastic partial differential equation and of the mathematical expectation can be exploited to show that the expectation of the square integrable random solution satisfies the corresponding deterministic, parabolic evolution equation. In the present paper we show that the covariance of the square integrable random solution of a parabolic stochastic partial differential equation satisfies a deterministic, tensorized evolution equation with a measure-valued, nonseparable forcing term. We establish the well-posedness of this equation in tensor products of Bochner spaces via a novel, tensorized space-time variational formulation of this evolution equation. This variational formulation, while being of interest in its own right, can serve as starting point for space-time compressive, adaptive Galerkin discretization techniques as outlined in $[1,7,8]$. The solution of the linear stochastic heat equation with Gaussian initial value is a Gaussian process. Therefore the knowledge of the covariance is of importance, since, together with the mean, it characterizes the law of the solution.

The outline of this paper is as follows: In Sect. 2 we introduce the required notation, and recapitulate basic results which are needed in the sequel. We review, in particular, the space-time variational formulation of linear, parabolic evolution problems from [9], and show how this can be tensorized by taking the tensor product of two such problems. In Sect. 3 we review the theory of stochastic partial differential equations of Itô type as stochastic differential equations in Hilbert spaces as presented in [3]. In Sect. 4 we first present a tensorized, linear evolution equation for the second moment of the solution of a random evolution partial differential equation. We then state and prove the main result that the covariance of the mild solution of the stochastic parabolic partial differential equation driven by $Q$-Wiener noise can be obtained as a weak, variational solution of a tensorized, deterministic parabolic evolution problem.

\section{Variational formulation of tensorized partial differential equations}

Let us review weak variational formulations of partial differential equations and solutions of tensorized equations in this section. Accordingly, we let $\left(H,\langle\cdot, \cdot\rangle_{H}\right)$ denote a separable real Hilbert space and $A: \mathcal{D}(A) \subset H \rightarrow H$ be a linear operator, which we assume to be densely defined, self-adjoint, positive definite, and not necessarily bounded but with compact inverse. Then there exists an increasing sequence of real numbers $\left(\alpha_{k}, k \in \mathbb{N}\right)$, which tends to infinity, and an orthonormal basis $\left(e_{k}, k \in \mathbb{N}\right)$ of $H$ such that $A e_{k}=\alpha_{k} e_{k}$. The domain of $A$ is characterized by

$$
\mathcal{D}(A):=\left\{\phi \in H, \sum_{k=1}^{\infty} \alpha_{k}^{2}\left\langle\phi, e_{k}\right\rangle_{H}^{2}<+\infty\right\} .
$$

Furthermore, $-A$ is the generator of an analytic semigroup of contractions $S=\left(S(t), t \in \mathbb{R}_{+}\right)$and we are able to define the square root of the operator $A$. 
This operator $A^{1 / 2}: \mathcal{D}\left(A^{1 / 2}\right) \rightarrow H$ is given by

$$
A^{1 / 2} \phi:=\sum_{k=1}^{\infty} \alpha_{k}^{1 / 2}\left\langle\phi, e_{k}\right\rangle_{H} e_{k}
$$

for all $\phi \in \mathcal{D}\left(A^{1 / 2}\right)$, where

$$
\mathcal{D}\left(A^{1 / 2}\right):=\left\{\phi \in H,\|\phi\|_{V}^{2}:=\sum_{k=1}^{\infty} \alpha_{k}\left\langle\phi, e_{k}\right\rangle_{H}^{2}<+\infty\right\}
$$

and $V:=\mathcal{D}\left(A^{1 / 2}\right)$ together with the norm $\|\cdot\|_{V}$ becomes a Hilbert space. The norm satisfies that $\|\phi\|_{V}=\left\|A^{1 / 2} \phi\right\|_{H}$, for all $\phi \in V$. Let us define the bilinear form $a: V \times V \rightarrow \mathbb{R}$ by

$$
a(\phi, \psi):=\left\langle A^{1 / 2} \phi, A^{1 / 2} \psi\right\rangle_{H},
$$

for all $\phi, \psi \in V$. It is symmetric, continuous, coercive, and injective. In the following, let $V^{*}$ denote the dual of $V$. By the Riesz representation theorem we identify $H$ with its dual and have the Gelfand triplet $V \subset H \cong H^{*} \subset V^{*}$. Then the linear operator $A: \mathcal{D}(A) \subset H \rightarrow H$ can be interpreted as a bounded linear operator $A: V \rightarrow V^{*}, A \in L\left(V ; V^{*}\right)$, via the bilinear form

$$
V^{*}\langle A \phi, \psi\rangle_{V}=a(\phi, \psi)={ }_{V}\left\langle\phi, A^{*} \psi\right\rangle_{V^{*}},
$$

for $\phi, \psi \in V$, where $V^{*}\langle\cdot, \cdot\rangle_{V}$ denotes the dual pairing between $V$ and $V^{*}$. Note that, although we assume that $A=A^{*}$ is self-adjoint, here and below we write $A^{*}$ when the operator appears as an adjoint operator. The operator $A \in L\left(V ; V^{*}\right)$ is boundedly invertible by the properties of $a$ and the Lax-Milgram lemma, and its norm is bounded by

$$
\|A\|_{L\left(V ; V^{*}\right)} \leq 1
$$

Let us fix the time interval $\mathbb{T}:=[0, T]$, for some $T<+\infty$, and define the Hilbert spaces

$$
\mathcal{X}:=L^{2}(\mathbb{T} ; V) \text { and } \mathcal{Y}:=L^{2}(\mathbb{T} ; V) \cap H_{0,\{T\}}^{1}\left(\mathbb{T} ; V^{*}\right)
$$

where

$$
H_{0,\{T\}}^{1}\left(\mathbb{T} ; V^{*}\right):=\left\{\phi \in H^{1}\left(\mathbb{T} ; V^{*}\right), \phi(T)=0\right\} .
$$

Let $\mathcal{X}^{*}$ and $\mathcal{Y}^{*}$ denote the adjoint spaces with respect to the pivot space $L^{2}(\mathbb{T} ; H)$, i.e.,

$$
\mathcal{X}^{*}=L^{2}\left(\mathbb{T} ; V^{*}\right) \text { and } \mathcal{Y}^{*}=L^{2}\left(\mathbb{T} ; V^{*}\right)+H^{-1}(\mathbb{T} ; V)
$$


In this framework, let us consider the parabolic partial differential equation

$$
\left(\partial_{t}+A\right) u=f
$$

with initial condition $u(0)=u_{0} \in H$, i.e., we want to solve the weak variational problem to find, for given $u_{0} \in H$ and $f \in \mathcal{Y}^{*}$, an element $u \in \mathcal{X}$ such that for all $v \in \mathcal{Y}$

$$
\mathcal{B}(u, v)=\mathcal{Y}^{*}\langle f, v\rangle_{\mathcal{Y}}+\left\langle u_{0}, v(0)\right\rangle_{H},
$$

where

$$
\begin{aligned}
\mathcal{B}(u, v) & :=\int_{0}^{T}\left({ }_{V}\left\langle u(t),-\partial_{t} v(t)\right\rangle_{V^{*}}+a(u(t), v(t))\right) \mathrm{d} t \\
& =\int_{0}^{T}\left\langle u(t),\left(-\partial_{t}+A^{*}\right) v(t)\right\rangle_{V^{*}} \mathrm{~d} t .
\end{aligned}
$$

It is shown in Theorem 2.3 in [9] that the variational problem (2.2) admits a unique solution $u \in \mathcal{X}$ and that the operator $B:=\partial_{t}+A: \mathcal{X} \rightarrow \mathcal{Y}^{*}$ is an isomorphism.

Let us consider the tensor spaces

$$
\mathcal{X}^{(2)}:=\mathcal{X} \otimes \mathcal{X} \cong L^{2}(\mathbb{T} ; \mathbb{R})^{(2)} \tilde{\otimes} V^{(2)}
$$

and

$$
\mathcal{Y}^{(2)}:=\mathcal{Y} \otimes \mathcal{Y}
$$

Since $\left(\mathcal{X}^{(2)}\right)^{*}=\left(\mathcal{X}^{*}\right)^{(2)}$, we use the abbreviation $\mathcal{X}^{(2) *}$ and we similarly define $\mathcal{Y}^{(2) *}$, which can be rewritten as

$$
\begin{aligned}
\mathcal{Y}^{(2) *}= & \left(L^{2}\left(\mathbb{T} ; V^{*}\right)+H^{-1}(\mathbb{T} ; V)\right)^{(2)} \\
\cong & L^{2}(\mathbb{T} ; \mathbb{R})^{(2)} \tilde{\otimes} V^{(2) *}+H^{-1}(\mathbb{T} ; \mathbb{R})^{(2)} \tilde{\otimes} V^{(2)} \\
& +L^{2}\left(\mathbb{T} ; V^{*}\right) \otimes H^{-1}(\mathbb{T} ; V)+H^{-1}(\mathbb{T} ; V) \otimes L^{2}\left(\mathbb{T} ; V^{*}\right) .
\end{aligned}
$$

Here, we denote by $\tilde{\otimes}$ the tensor product which separates the spaces with respect to time and space. Let us define the tensorized bilinear form

$$
\begin{aligned}
\mathcal{B}^{(2)}(u, v) & :=\int_{0}^{T} \int_{0}^{T} V^{(2)}\left\langle u\left(t, t^{\prime}\right),\left(-\partial_{t}+A^{*}\right)^{(2)} v\left(t, t^{\prime}\right)\right\rangle_{V^{(2) *}} \mathrm{~d} t \mathrm{~d} t^{\prime} \\
& ={ }_{\mathcal{X}^{(2)}}\left\langle u,\left(-\partial_{t}+A^{*}\right)^{(2)} v\right\rangle_{\mathcal{X}^{(2) *}},
\end{aligned}
$$


for all $u \in \mathcal{X}^{(2)}$ and $v \in \mathcal{Y}^{(2)}$. Then since $B=\partial_{t}+A$ is an isomorphism so is $B^{(2)}$, and the weak variational problem to find, for given $u_{0} \in H^{(2)}$ and $f \in \mathcal{Y}^{(2) *}$, an element $u \in \mathcal{X}^{(2)}$ such that

$$
\mathcal{B}^{(2)}(u, v)=\mathcal{Y}^{(2) *}\langle f, v\rangle_{\mathcal{Y}^{(2)}}+\left\langle u_{0}, v(0,0)\right\rangle_{H^{(2)}},
$$

for all $v \in \mathcal{Y}^{(2)}$, admits a unique solution. The corresponding strong form of the tensorized partial differential equation (2.3) reads

$$
\begin{aligned}
\left(\partial_{t}+A\right)^{(2)} u & =f \\
\left(\partial_{t}+A\right) \otimes I u(\cdot, 0) & =0 \\
I \otimes\left(\partial_{t}+A\right) u(0, \cdot) & =0 \\
u(0,0) & =u_{0} .
\end{aligned}
$$

In Sect. 4 we will show that second moments and covariances of stochastic and random partial differential equations are solutions of the tensorized partial differential equation (2.3) with $u_{0}$ and $f$ chosen appropriately.

\section{Stochastic partial differential equations}

In this section we consider stochastic partial differential equations and their mild solutions in the framework of [3] and [5].

Let $\left(\Omega, \mathcal{A},\left(\mathcal{F}_{t}\right)_{t \geq 0}, P\right)$ be a filtered probability space that satisfies the "usual conditions". Furthermore, let $\left(H,\langle\cdot, \cdot\rangle_{H}\right)$ be a separable real Hilbert space with corresponding norm denoted by $\|\cdot\|_{H}$. The Lebesgue-Bochner space $L^{2}(\Omega ; H)$ is the space of all square integrable, $H$-valued random variables, i.e., the space of all $H$-valued random variables $X$ such that

$$
\|X\|_{L^{2}(\Omega ; H)}^{2}:=\mathbb{E}\left[\|X\|_{H}^{2}\right]<+\infty .
$$

For all $X \in L^{2}(\Omega ; H)$, the second moment of $X$

$$
\mathbb{M}^{(2)} X:=\mathbb{E}\left[X^{(2)}\right]=\mathbb{E}[X \otimes X]
$$

is well-defined as an element of $H^{(2)}$ since

$$
\left\|\mathbb{M}^{(2)} X\right\|_{H^{(2)}} \leq \mathbb{E}\left[\left\|X^{(2)}\right\|_{H^{(2)}}\right]=\mathbb{E}\left[\|X\|_{H}^{2}\right]=\|X\|_{L^{2}(\Omega ; H)}^{2} .
$$

Furthermore, we define the covariance $\operatorname{Cov}(X)$ of $X \in L^{2}(\Omega ; H)$ as element of $H^{(2)}$ by

$$
\operatorname{Cov}(X):=\mathbb{M}^{(2)}(X-\mathbb{E}[X])
$$

i.e., $\operatorname{Cov}(X)$ is the centered second moment of $X$. 
Let us denote by $L_{\mathrm{N}}^{+}(H)$ the space of all nonnegative, symmetric, nuclear operators on $H$. Then, for $X \in L^{2}(\Omega ; H)$, the covariance $\operatorname{Cov}(X)$ corresponds in a one-to-one fashion to a unique operator $Q_{X} \in L_{\mathrm{N}}^{+}(H)$ given by the relation

$$
\langle\operatorname{Cov}(X), \varphi \otimes \psi\rangle_{H^{(2)}}=\left\langle Q_{X} \varphi, \psi\right\rangle_{H},
$$

for all $\varphi, \psi \in H$. We shall refer to the operator $Q_{X}$ as the covariance operator of $X$.

For $Q \in L_{\mathrm{N}}^{+}(H)$, the $H$-valued stochastic process $W:=\left(W(t), t \in \mathbb{R}_{+}\right)$is called a $Q$-Wiener process if it starts in zero $P$-almost surely, it has $P$-almost surely continuous trajectories, the increments are independent, and for $s<t$, the increment $W(t)-W(s)$ is Gaussian distributed with expectation zero and covariance operator $(t-s) Q$. Let us denote by $q$ the covariance in $H^{(2)}$ that corresponds to $Q$, i.e., $q$ is uniquely defined by the condition

$$
\langle q, \varphi \otimes \psi\rangle_{H^{(2)}}=\langle Q \varphi, \psi\rangle_{H},
$$

for all $\varphi, \psi \in H$. Since $Q \in L_{\mathrm{N}}^{+}(H)$, there exists an orthonormal basis $\left(e_{n}, n \in \mathbb{N}\right)$ of $H$ consisting of eigenvectors of $Q$. Therefore, we have the representation $Q e_{n}=\gamma_{n} e_{n}$, where $\gamma_{n} \geq 0$ is the eigenvalue corresponding to $e_{n}$, for $n \in \mathbb{N}$. Then the square root of $Q$ is defined as

$$
Q^{1 / 2} \psi:=\sum_{n \in \mathbb{N}} \gamma_{n}^{1 / 2}\left\langle\psi, e_{n}\right\rangle_{H} e_{n}
$$

for $\psi \in H$, and $Q^{-1 / 2}$ denotes the pseudo inverse of $Q^{1 / 2}$. Let us denote by $\left(\mathcal{H},\langle\cdot, \cdot\rangle_{\mathcal{H}}\right)$ the Hilbert space defined by $\mathcal{H}:=Q^{1 / 2}(H)$ endowed with the inner product $\langle\psi, \phi\rangle_{\mathcal{H}}:=\left\langle Q^{-1 / 2} \psi, Q^{-1 / 2} \phi\right\rangle_{H}$, for $\psi, \phi \in \mathcal{H}$. Let $L_{\mathrm{HS}}(\mathcal{H} ; H)$ refer to the space of all Hilbert-Schmidt operators from $\mathcal{H}$ to $H$, and by $\|\cdot\|_{L_{\mathrm{HS}}(\mathcal{H} ; H)}$ we denote the corresponding norm. It holds that $q$ has an expansion with respect to the eigenvalues and eigenvectors of $Q$ given by

$$
q=\sum_{n \in \mathbb{N}} \gamma_{n} e_{n} \otimes e_{n}
$$

Furthermore, $W$ admits a Karhunen-Loève expansion, i.e., for all $t \in \mathbb{T}$, it holds that

$$
W(t)=\sum_{n \in \mathbb{N}}\left\langle W(t), e_{n}\right\rangle_{H} e_{n}=\sum_{n \in \mathbb{N}} \gamma_{n}^{1 / 2} W_{n}(t) e_{n},
$$

where $\left(W_{n}, n \in \mathbb{N}\right)$ is a sequence of independent, real-valued Brownian motions.

For $t \in \mathbb{T}$, let us denote by

$$
\int_{0}^{t} \Phi(s) \mathrm{d} W(s)
$$


the stochastic integral with respect to $\Phi \in \mathbb{L}_{\mathcal{H}, \mathbb{T}}^{2}(H)$ with

$$
\mathbb{E}\left[\int_{0}^{T}\|\Phi(s)\|_{L_{\mathrm{HS}}(\mathcal{H} ; H)}^{2} \mathrm{~d} s\right]<+\infty .
$$

Here, $\mathbb{L}_{\mathcal{H}, \mathbb{T}}^{2}(H):=L^{2}\left(\Omega \times \mathbb{T} ; L_{\mathrm{HS}}(\mathcal{H} ; H)\right)$ denotes the space of integrands with respect to the measure space $\left(\Omega \times \mathbb{T}, \mathcal{P}_{\mathbb{T}}, P \otimes \lambda\right)$, where $\mathcal{P}_{\mathbb{T}}$ is the $\sigma$-algebra of predictable sets in $\Omega \times \mathbb{T}$ and $\lambda$ denotes the Lebesgue measure.

Then the stochastic integral satisfies the Itô isometry

$$
\mathbb{E}\left[\left\|\int_{0}^{t} \Phi(s) \mathrm{d} W(s)\right\|_{H}^{2}\right]=\mathbb{E}\left[\int_{0}^{t}\|\Phi(s)\|_{L_{\mathrm{HS}}(\mathcal{H} ; H)}^{2} \mathrm{~d} s\right],
$$

for all $t \in \mathbb{T}$. For an introduction to stochastic integrals with respect to Hilbert-spacevalued stochastic processes, the reader is referred to $[2,3,5,6]$.

We define the weak stochastic integral by

$$
\int_{0}^{t}\langle\Psi(s), \Phi(s) \mathrm{d} W(s)\rangle_{H}:=\int_{0}^{t} \tilde{\Phi}_{\Psi}(s) \mathrm{d} W(s),
$$

for $t \in \mathbb{T}$, with $\tilde{\Phi}_{\Psi}(s) \in \mathcal{H}^{*}, s \in \mathbb{T}$, such that for all $u \in \mathcal{H}$

$$
\tilde{\Phi}_{\Psi}(s) u:=\langle\Psi(s), \Phi(s) u\rangle_{H},
$$

where $\Psi$ is an $H$-valued, continuous, adapted stochastic process and $\Phi \in \mathbb{L}_{\mathcal{H}, \mathbb{T}}^{2}(H)$. The weak stochastic integral is well-defined (cf. [5]). Since the stochastic integral is a martingale (cf. Theorem 4.12 in [3]), it satisfies for $t \in \mathbb{T}$ that

$$
\mathbb{E}\left[\int_{0}^{t}\langle\Psi(s), \Phi(s) \mathrm{d} W(s)\rangle_{H} \mid \mathcal{F}_{0}\right]=\mathbb{E}\left[\int_{0}^{t} \tilde{\Phi}_{\Psi}(s) \mathrm{d} W(s) \mid \mathcal{F}_{0}\right]=0 .
$$

Furthermore, the weak stochastic integral satisfies a general Itô isometry, which is shown in the following lemma.

Lemma 3.1 Let $v_{1}, v_{2} \in C^{0}(\mathbb{T} ; H)$ and $\Phi \in \mathbb{L}_{\mathcal{H}, \mathbb{T}}^{2}(H)$. Then the weak stochastic integral satisfies

$$
\begin{aligned}
\mathbb{E}\left[\int_{0}^{t}\left\langle v_{1}(s), \Phi(s) \mathrm{d} W(s)\right\rangle_{H} \int_{0}^{t}\left\langle v_{2}\left(s^{\prime}\right), \Phi\left(s^{\prime}\right) \mathrm{d} W\left(s^{\prime}\right)\right\rangle_{H}\right] \\
=\int_{0}^{t}\left\langle v_{1}(s) \otimes v_{2}(s), \mathbb{E}[\Phi(s) \otimes \Phi(s)] q\right\rangle_{H^{(2)}} \mathrm{d} s .
\end{aligned}
$$


Proof Let $t \in \mathbb{T}$ be fixed. Using the Karhunen-Loève expansion of $W$, we rewrite the weak stochastic integral

$$
\int_{0}^{t}\left\langle v_{1}(s), \Phi(s) \mathrm{d} W(s)\right\rangle_{H}=\sum_{n=1}^{\infty} \gamma_{n}{ }^{1 / 2} \int_{0}^{t}\left\langle v_{1}(s), \Phi(s) e_{n}\right\rangle_{H} \mathrm{~d} W_{n}(s) .
$$

Then the independence as well as the martingale property of the real-valued Wiener processes $W_{n}, n \in \mathbb{N}$, implies that

$$
\begin{aligned}
& \mathbb{E}\left[\int_{0}^{t}\left\langle v_{1}(s), \Phi(s) \mathrm{d} W(s)\right\rangle_{H} \int_{0}^{t}\left\langle v_{2}\left(s^{\prime}\right), \Phi\left(s^{\prime}\right) \mathrm{d} W\left(s^{\prime}\right)\right\rangle_{H}\right] \\
& =\sum_{n, m=1}^{\infty} \gamma_{n}^{1 / 2} \gamma_{m}^{1 / 2} \mathbb{E}\left[\int_{0}^{t}\left\langle v_{1}(s), \Phi(s) e_{n}\right\rangle_{H} \mathrm{~d} W_{n}(s) \int_{0}^{t}\left\langle v_{2}\left(s^{\prime}\right), \Phi\left(s^{\prime}\right) e_{m}\right\rangle_{H} \mathrm{~d} W_{m}\left(s^{\prime}\right)\right] \\
& =\sum_{n=1}^{\infty} \gamma_{n} \mathbb{E}\left[\int_{0}^{t}\left\langle v_{1}(s), \Phi(s) e_{n}\right\rangle_{H} \mathrm{~d} W_{n}(s) \int_{0}^{t}\left\langle v_{2}\left(s^{\prime}\right), \Phi\left(s^{\prime}\right) e_{n}\right\rangle_{H} \mathrm{~d} W_{n}\left(s^{\prime}\right)\right] \\
& =\sum_{n=1}^{\infty} \gamma_{n} \mathbb{E}\left[\int_{0}^{t}\left\langle v_{1}(s), \Phi(s) e_{n}\right\rangle_{H}\left\langle v_{2}(s), \Phi(s) e_{n}\right\rangle_{H} \mathrm{~d} s\right]
\end{aligned}
$$

where we applied an Itô isometry for real-valued Wiener processes (see Proposition 1.2 in [4]) in the last step. By the definition of the tensor product and of $q$ in (3.1), we get that

$$
\begin{aligned}
& \sum_{n=1}^{\infty} \gamma_{n} \mathbb{E}\left[\int_{0}^{t}\left\langle v_{1}(s), \Phi(s) e_{n}\right\rangle_{H}\left\langle v_{2}(s), \Phi(s) e_{n}\right\rangle_{H} \mathrm{~d} s\right] \\
& \quad=\int_{0}^{t} \mathbb{E}\left[\left\langle v_{1}(s) \otimes v_{2}(s), \sum_{n=1}^{\infty} \gamma_{n}\left(\Phi(s) e_{n}\right) \otimes\left(\Phi(s) e_{n}\right)\right\rangle_{H^{(2)}} \mathrm{d} s\right] \\
& =\int_{0}^{t}\left\langle v_{1}(s) \otimes v_{2}(s), \mathbb{E}[\Phi(s) \otimes \Phi(s)] q\right\rangle_{H^{(2)}} \mathrm{d} s,
\end{aligned}
$$

which finishes the proof.

Having introduced some properties of Hilbert-space-valued random variables and the stochastic integral with respect to a $Q$-Wiener process, we start the discussion of the stochastic partial differential equation

$$
\mathrm{d} X(t)+A X(t) \mathrm{d} t=\mathrm{d} W(t)
$$


with $\mathcal{F}_{0}$-measurable initial condition $X(0)=X_{0} \in L^{2}(\Omega ; H)$, for $t \in \mathbb{T}$. Here, $W$ is a $Q$-Wiener process and $A$ satisfies the assumptions that were made in Sect. 2, i.e., $A$ is a linear operator that is densely defined, self-adjoint, positive definite, and not necessarily bounded but with compact inverse. Then (3.4) admits a unique mild solution $X$, i.e., $X$ is a predictable process that satisfies $\sup _{t \in \mathbb{T}}\|X(t)\|_{L^{2}(\Omega ; H)}<+\infty$ and

$$
X(t)=S(t) X_{0}+\int_{0}^{t} S(t-s) \mathrm{d} W(s)
$$

for all $t \in \mathbb{T}$. Furthermore, this solution is equal to the unique weak solution, i.e., $X$ is a predictable process that satisfies $\sup _{t \in \mathbb{T}}\|X(t)\|_{L^{2}(\Omega ; H)}<+\infty$ and for all $\varphi \in \mathcal{D}\left(A^{*}\right)$ and $t \in \mathbb{T}$

$$
\langle\varphi, X(t)\rangle_{H}=\left\langle\varphi, X_{0}\right\rangle_{H}-\int_{0}^{t}\left\langle A^{*} \varphi, X(s)\right\rangle_{H} \mathrm{~d} s+\langle\varphi, W(t)\rangle_{H} .
$$

Recall that, although $A=A^{*}$ is self-adjoint, we write $A^{*}$ when the operator appears as an adjoint operator.

The solution $X$ of (3.4) has certain properties. In the following lemma, we establish another weak formulation. There we use the space of test functions

$$
C_{0,\{T\}}^{1}\left(\mathbb{T} ; \mathcal{D}\left(A^{*}\right)\right):=\left\{\phi \in C^{1}\left(\mathbb{T} ; \mathcal{D}\left(A^{*}\right)\right), \phi(T)=0\right\} .
$$

Lemma 3.2 Let $X$ be the mild solution of the stochastic partial differential equation (3.4). Then $X$ satisfies for all $\varphi \in C_{0,\{T\}}^{1}\left(\mathbb{T} ; \mathcal{D}\left(A^{*}\right)\right) P$-almost surely that

$$
\left\langle X,\left(-\partial_{t}+A^{*}\right) \varphi\right\rangle_{L^{2}(\mathbb{T} ; H)}=\left\langle X_{0}, \varphi(0)\right\rangle_{H}+\int_{0}^{T}\langle\varphi(t), \mathrm{d} W(t)\rangle_{H} .
$$

Proof The lemma is a direct consequence of (9.20) in [5], where we use that $\varphi(T)=0$ by definition.

Furthermore, we prove regularity of the second moment of the solution.

Lemma 3.3 The second moment $\mathbb{M}^{(2)} X$ of the mild solution $X$ of (3.4) is an element of $\mathcal{X}^{(2)}$.

Proof We first observe that

$$
\left\|\mathbb{M}^{(2)} X\right\|_{\mathcal{X}^{(2)}} \leq \mathbb{E}\left[\left\|X^{(2)}\right\|_{\mathcal{X}^{(2)}}\right]=\mathbb{E}\left[\|X\|_{\mathcal{X}}^{2}\right]
$$

Using the definition of the norm and the independence of the stochastic integral of $\mathcal{F}_{0}$, we obtain that 


$$
\begin{aligned}
\mathbb{E}\left[\|X\|_{\mathcal{X}}^{2}\right] & =\int_{0}^{T} \mathbb{E}\left[\left\|A^{1 / 2} S(t) X_{0}+A^{1 / 2} \int_{0}^{t} S(t-s) \mathrm{d} W(s)\right\|_{H}^{2}\right] \mathrm{d} t \\
& =\mathbb{E}\left[\int_{0}^{T}\left\|A^{1 / 2} S(t) X_{0}\right\|_{H}^{2} \mathrm{~d} t\right]+\int_{0}^{T} \mathbb{E}\left[\left\|\int_{0}^{t} A^{1 / 2} S(t-s) \mathrm{d} W(s)\right\|_{H}^{2}\right] \mathrm{d} t .
\end{aligned}
$$

For the first term, we use that, for $v \in H$,

$$
\int_{0}^{T}\left\|A^{1 / 2} S(t) v\right\|_{H}^{2} \mathrm{~d} t \leq \frac{1}{2}\|v\|_{H}^{2},
$$

which is easily proved by the spectral representation of $S(t)$. Hence, the first term is bounded by $\frac{1}{2}\left\|X_{0}\right\|_{L^{2}(\Omega ; H)}^{2}$. To the second term, we apply the Itô isometry (3.2), the definition of the Hilbert-Schmidt norm, and again (3.5) to get

$$
\begin{aligned}
\mathbb{E}\left[\left\|\int_{0}^{t} A^{1 / 2} S(t-s) \mathrm{d} W(s)\right\|_{H}^{2}\right] & =\int_{0}^{t}\left\|A^{1 / 2} S(t-s)\right\|_{L_{\mathrm{HS}}(\mathcal{H} ; H)}^{2} \mathrm{~d} s \\
& =\sum_{n \in \mathbb{N}} \gamma_{n} \int_{0}^{t}\left\|A^{1 / 2} S(t-s) e_{n}\right\|_{H}^{2} \mathrm{~d} s \leq \frac{1}{2} \operatorname{Tr}(Q) .
\end{aligned}
$$

This leads to

$$
\left\|\mathbb{M}^{(2)} X\right\|_{\mathcal{X}^{(2)}} \leq \frac{1}{2}\left(\left\|X_{0}\right\|_{L^{2}(\Omega ; H)}^{2}+T \operatorname{Tr}(Q)\right)<+\infty
$$

by our assumptions on $X_{0}$ and $Q$.

\section{Covariance partial differential equation}

In this section we calculate second moments and covariances of two different classes of stochastic evolution partial differential equations. First, we look at random partial differential equations, where the initial condition and the right hand side of (2.1) are random variables. In a second step we deal with mild solutions of the parabolic stochastic partial differential equation (3.4). We show that second moments and covariances of the equations are equal to the unique solution of the tensorized partial differential equation (2.3), where the parameters $u_{0}$ and $f$ in (2.3) have to be chosen according to the random or stochastic partial differential equation.

\subsection{Random partial differential equation}

In the framework of Sect. 2, let us consider in a first step the random partial differential equation 


$$
\left(\partial_{t}+A\right) U=F
$$

on the time interval $\mathbb{T}$ with initial condition $U(0)=U_{0} \in L^{2}(\Omega ; H)$ and $F \in$ $L^{2}\left(\Omega ; \mathcal{Y}^{*}\right)$. For almost every fixed $\omega \in \Omega$, it holds that $U_{0}(\omega) \in H$ and $F(\omega) \in \mathcal{Y}^{*}$ and it was shown in Sect. 2 that the weak variational problem

$$
\mathcal{B}(u, v)={ }_{\mathcal{Y}^{*}}\langle F(\omega), v\rangle_{\mathcal{Y}}+\left\langle U_{0}(\omega), v(0)\right\rangle_{H}
$$

has a unique solution $u \in \mathcal{X}$ that depends on $\omega$ and that we denote by $U(\omega):=u$. Furthermore, since $\partial_{t}+A$ is an isomorphism with bounded inverse, $U$ is an $\mathcal{X}$-valued random variable that is in $L^{2}(\Omega ; \mathcal{X})$ by the properties of $U_{0}$ and $F$. Therefore, $U$ is a solution of (4.1) in the sense that $U \in L^{2}(\Omega ; \mathcal{X})$ and $U(\omega)$ satisfies the weak variational problem (4.2) for almost every $\omega \in \Omega$.

Since $U \in L^{2}(\Omega ; \mathcal{X})$, the second moment $\mathbb{M}^{(2)} U$ of the variational solution $U$ of the random partial differential equation (4.1) is well-defined. Furthermore, $\mathbb{M}^{(2)} U$ satisfies for $v_{1}, v_{2} \in \mathcal{Y}$ that

$$
\begin{aligned}
\mathcal{B}^{(2)} & \left.\mathbb{M}^{(2)} U, v_{1} \otimes v_{2}\right)=\mathbb{E}\left[\mathcal{B}\left(U, v_{1}\right) \mathcal{B}\left(U, v_{2}\right)\right] \\
= & \mathbb{E}\left[\left(\mathcal{Y}^{*}\left\langle F, v_{1}\right\rangle_{\mathcal{Y}}+\left\langle U_{0}, v_{1}(0)\right\rangle_{H}\right)\left(\mathcal{Y}^{*}\left\langle F, v_{2}\right\rangle_{\mathcal{Y}}+\left\langle U_{0}, v_{2}(0)\right\rangle_{H}\right)\right] \\
= & \mathcal{Y}^{(2) *}\left\langle\mathbb{M}^{(2)} F, v_{1} \otimes v_{2}\right\rangle_{\mathcal{Y}^{(2)}}+\left\langle\mathbb{M}^{(2)} U_{0},\left(v_{1} \otimes v_{2}\right)(0,0)\right\rangle_{H^{(2)}} \\
& +\mathbb{E}\left[\mathcal{Y}^{*}\left\langle F, v_{1}\right\rangle_{\mathcal{Y}}\left\langle U_{0}, v_{2}(0)\right\rangle_{H}\right]+\mathbb{E}\left[\left\langle U_{0}, v_{1}(0)\right\rangle_{H} \mathcal{Y}^{*}\left\langle F, v_{2}\right\rangle_{\mathcal{Y}}\right] .
\end{aligned}
$$

The two summands in the last line will be equal to zero if we assume that $F$ and $U_{0}$ are independent and that $\mathbb{E}(F)=0$ or $\mathbb{E}\left(U_{0}\right)=0$. This implies that $\mathbb{M}^{(2)} U$ solves the weak variational problem (2.3) with $u_{0}:=\mathbb{M}^{(2)} U_{0}$ and $f:=\mathbb{M}^{(2)} F$, if $F$ and $U_{0}$ are independent and if $\mathbb{E}(F)=0$ or $\mathbb{E}\left(U_{0}\right)=0$.

Let us next look at the covariance $\operatorname{Cov}(U)=\mathbb{M}^{(2)}(U-\mathbb{E}[U])$ of the solution $U$ of (4.1). It satisfies for $v_{1}, v_{2} \in \mathcal{Y}$ that

$$
\begin{aligned}
\mathcal{B}^{(2)} & \left(\operatorname{Cov}(U), v_{1} \otimes v_{2}\right)=\mathbb{E}\left[\mathcal{B}\left(U-\mathbb{E}[U], v_{1}\right) \mathcal{B}\left(U-\mathbb{E}[U], v_{2}\right)\right] \\
= & \mathcal{Y}^{(2) *}\left\langle\operatorname{Cov}(F), v_{1} \otimes v_{2}\right\rangle_{\mathcal{Y}^{(2)}}+\left\langle\operatorname{Cov}\left(U_{0}\right),\left(v_{1} \otimes v_{2}\right)(0,0)\right\rangle_{H^{(2)}} \\
& +\mathbb{E}\left[\mathcal{Y}^{*}\left\langle F-\mathbb{E}[F], v_{1}\right\rangle_{\mathcal{Y}}\left\langle U_{0}-\mathbb{E}\left[U_{0}\right], v_{2}(0)\right\rangle_{H}\right] \\
& +\mathbb{E}\left[\left\langle U_{0}-\mathbb{E}\left[U_{0}\right], v_{1}(0)\right\rangle_{H} \mathcal{Y}^{*}\left\langle F-\mathbb{E}[F], v_{2}\right\rangle_{\mathcal{Y}}\right],
\end{aligned}
$$

since

$$
\begin{aligned}
\mathcal{B}(\mathbb{E}[U], v)=\mathbb{E}[\mathcal{B}(U, v)] & =\mathbb{E}\left[\mathcal{Y}^{*}\langle F, v\rangle_{\mathcal{Y}}+\left\langle U_{0}, v(0)\right\rangle_{H}\right] \\
& ={ }_{\mathcal{Y}^{*}}\langle\mathbb{E}[F], v\rangle_{\mathcal{Y}}+\left\langle\mathbb{E}\left[U_{0}\right], v(0)\right\rangle_{H},
\end{aligned}
$$

for all $v \in \mathcal{Y}$. Therefore, $\operatorname{Cov}(U)$ solves the weak variational problem (2.3) with $u_{0}:=\operatorname{Cov}\left(U_{0}\right)$ and $f:=\operatorname{Cov}(F)$, provided that $F$ and $U_{0}$ are independent.

We have thus seen that, under additional assumptions concerning the independence and the expectation of the data $U_{0}$ and $F$, the second moment and the covariance of the random partial differential equation (4.1) are the unique solutions of the tensorized 
partial differential equation (2.3) with appropriately chosen data $u_{0}$ and $f$. We will see in the next section that such assumptions are naturally met by the data of the stochastic partial differential equation (3.4).

\subsection{Stochastic partial differential equation}

In this subsection we consider the second moment $\mathbb{M}^{(2)} X$ of the mild solution $X$ of the stochastic partial differential equation (3.4) with initial condition $X_{0} \in L^{2}(\Omega ; H)$ and show that it is equal to the unique solution of the weak variational problem (2.3), where $u_{0}=\mathbb{M}^{(2)} X_{0}$ and $f=\delta \tilde{\otimes} q$. Here, $\delta$ is a Dirac distribution $\delta$ to be introduced next.

To describe the temporal correlation of the $Q$-Wiener process $W$, we define the distribution $\delta$ in the sense of L. Schwartz as functional acting on test functions $\phi \in C_{0}^{\infty}\left(\mathbb{T}^{2} ; \mathbb{R}\right)$ by

$$
\langle\delta, \phi\rangle_{L^{2}(\mathbb{T} ; \mathbb{R})^{(2)}}=\int_{0}^{T} \phi(s, s) \mathrm{d} s
$$

Therefore, $\delta$ is a measure which is supported on the diagonal of $\mathbb{T}^{2}$. By Lemma 3 in [10], $\delta \in H^{-s,-s}\left(\mathbb{T}^{2} ; \mathbb{R}\right) \cong H^{-s}(\mathbb{T} ; \mathbb{R})^{(2)}$, for $s>1 / 4$. In the following lemma we prove that an additional spatial regularity assumption on the covariance operator $Q$ combined with the low temporal regularity of the Wiener process implies that the tensor product $\delta \tilde{\otimes} q$ is in $H^{-1}(\mathbb{T} ; V)^{(2)}$ and therefore in $\mathcal{Y}^{(2) *}$.

Lemma 4.1 Let $\operatorname{Tr}(A Q)<+\infty$. Then $\delta \tilde{\otimes} q \in \mathcal{Y}^{(2) *}$.

Proof We first remark that $\operatorname{Tr}(A Q)<+\infty$ implies by (3.1) that

$$
\|q\|_{V^{(2)}}=\left\|\sum_{n=1}^{\infty} \gamma_{n} e_{n} \otimes e_{n}\right\|_{V^{(2)}} \leq \sum_{n=1}^{\infty} \gamma_{n}\left\|e_{n} \otimes e_{n}\right\|_{V^{(2)}}=\operatorname{Tr}(A Q)<+\infty
$$

and hence that $q \in V^{(2)}$. It remains to show that $\delta \in H^{-1}(\mathbb{T} ; \mathbb{R})^{(2)}$ to finish the proof. This is true since $\delta \in H^{-s}(\mathbb{T} ; \mathbb{R})^{(2)}$, for all $s>1 / 4$ by Lemma 3 in [10].

Having shown some regularity of the expression $\delta \tilde{\otimes} q$, we are now able to state and prove the main result of the paper.

Theorem 4.2 Let $X$ be the mild solution of the stochastic partial differential equation (3.4) with $\mathcal{F}_{0}$-measurable initial condition $X_{0} \in L^{2}(\Omega ; H)$. Moreover, assume that $\operatorname{Tr}(A Q)<+\infty$. Then the second moment $\mathbb{M}^{2} X$ solves the weak variational problem $(2.3)$ with $u_{0}:=\mathbb{M}^{(2)} X_{0}$ and $f:=\delta \tilde{\otimes} q$.

Proof First, we remark that the embedding $C_{0,\{T\}}^{1}\left(\mathbb{T} ; \mathcal{D}\left(A^{*}\right)\right) \subset \mathcal{Y}$ is continuous and dense. Therefore, it is sufficient to show (2.3) for $v_{1} \otimes v_{2}$ with 
$v_{1}, v_{2} \in C_{0,\{T\}}^{1}\left(\mathbb{T} ; \mathcal{D}\left(A^{*}\right)\right)$. So, let $v_{1}, v_{2} \in C_{0,\{T\}}^{1}\left(\mathbb{T} ; \mathcal{D}\left(A^{*}\right)\right)$. Then using the definition of the bilinear form $\mathcal{B}^{(2)}$, we obtain that

$$
\begin{aligned}
\mathcal{B}^{(2)}\left(\mathbb{M}^{2} X, v_{1} \otimes v_{2}\right) & ={ }_{\mathcal{X}}^{(2)}\left\langle\mathbb{M}^{2} X,\left(-\partial_{t}+A^{*}\right)^{(2)}\left(v_{1} \otimes v_{2}\right)\right\rangle_{\mathcal{X}^{(2) *}} \\
& =\mathbb{E}\left[\mathcal{X}_{\mathcal{X}}\left\langle X,\left(-\partial_{t}+A^{*}\right) v_{1}\right\rangle_{\mathcal{X}^{*}} \mathcal{X}\left\langle X,\left(-\partial_{t}+A^{*}\right) v_{2}\right\rangle_{\mathcal{X}^{*}}\right]
\end{aligned}
$$

Furthermore, the regularity of $v_{1}$ and $v_{2}$ implies that

$$
\begin{aligned}
& \mathbb{E}\left[\mathcal{X}\left\langle X,\left(-\partial_{t}+A^{*}\right) v_{1}\right\rangle_{\mathcal{X}^{*}}\left\langle\mathcal{X}\left\langle X,\left(-\partial_{t}+A^{*}\right) v_{2}\right\rangle_{\mathcal{X}^{*}}\right]\right. \\
& \quad=\mathbb{E}\left[\left\langle X,\left(-\partial_{t}+A^{*}\right) v_{1}\right\rangle_{L^{2}(\mathbb{T} ; H)}\left\langle X,\left(-\partial_{t}+A^{*}\right) v_{2}\right\rangle_{L^{2}(\mathbb{T} ; H)}\right]
\end{aligned}
$$

The application of Lemma 3.2 leads to

$$
\begin{aligned}
\mathbb{E}\left[\left\langle X,\left(-\partial_{t}+A^{*}\right) v_{1}\right\rangle_{L^{2}(\mathbb{T} ; H)}\left\langle X,\left(-\partial_{t}+A^{*}\right) v_{2}\right\rangle_{L^{2}(\mathbb{T} ; H)}\right] \\
=\mathbb{E}\left[\left\langle X_{0}, v_{1}(0)\right\rangle_{H}\left\langle X_{0}, v_{2}(0)\right\rangle_{H}\right]+\mathbb{E}\left[\left\langle X_{0}, v_{1}(0)\right\rangle_{H} \int_{0}^{T}\left\langle v_{2}(t), \mathrm{d} W(t)\right\rangle_{H}\right] \\
+\mathbb{E}\left[\int_{0}^{T}\left\langle v_{1}(t), \mathrm{d} W(t)\right\rangle_{H}\left\langle X_{0}, v_{2}(0)\right\rangle_{H}\right] \\
+\mathbb{E}\left[\int_{0}^{T}\left\langle v_{1}(t), \mathrm{d} W(t)\right\rangle_{H} \int_{0}^{T}\left\langle v_{2}(t), \mathrm{d} W(t)\right\rangle_{H}\right] .
\end{aligned}
$$

The second and third terms on the right hand side are equal to zero by (3.3) and since $X_{0}$ is $\mathcal{F}_{0}$-measurable. We apply Lemma 3.1 to the fourth term and the definition of the tensor product to the first and the fourth terms to derive

$$
\begin{aligned}
& \mathbb{E}\left[\left\langle X,\left(-\partial_{t}+A^{*}\right) v_{1}\right\rangle_{L^{2}(\mathbb{T} ; H)}\left\langle X,\left(-\partial_{t}+A^{*}\right) v_{2}\right\rangle_{L^{2}(\mathbb{T} ; H)}\right] \\
& \quad=\left\langle\mathbb{M}^{2} X_{0},\left(v_{1} \otimes v_{2}\right)(0,0)\right\rangle_{H^{(2)}}+\left\langle\delta \tilde{\otimes} q, v_{1} \otimes v_{2}\right\rangle_{L^{2}(\mathbb{T} ; H)^{(2)}} .
\end{aligned}
$$

The desired assertion now follows, since

$$
\left\langle\delta \tilde{\otimes} q, v_{1} \otimes v_{2}\right\rangle_{L^{2}(\mathbb{T} ; H)^{(2)}}=\mathcal{Y}^{(2) *}\left\langle\delta \tilde{\otimes} q, v_{1} \otimes v_{2}\right\rangle_{\mathcal{Y}^{(2)}},
$$

due to Lemma 4.1 and the properties of $v_{1}$ and $v_{2}$.

Corollary 4.3 Under the assumptions of Theorem 4.2, the covariance $\operatorname{Cov}(X)$ of the mild solution $X$ of (3.4) satisfies

$$
\mathcal{B}^{(2)}(\operatorname{Cov}(X), v)=\mathcal{Y}^{(2) *}\langle\delta \tilde{\otimes} q, v\rangle_{\mathcal{Y}^{(2)}}+\left\langle\operatorname{Cov}\left(X_{0}\right), v(0,0)\right\rangle_{H^{(2)}},
$$

for all $v \in \mathcal{Y}^{(2)}$, and therefore it solves the weak variational problem (2.3) with $u_{0}:=\operatorname{Cov}\left(X_{0}\right)$ and $f:=\delta \tilde{\otimes} q$. 
Proof For all $t \in \mathbb{T}$, it holds that

$$
\begin{aligned}
X(t)-\mathbb{E}[X(t)] & =S(t) X_{0}+\int_{0}^{t} S(t-s) \mathrm{d} W(s)-S(t) \mathbb{E}\left[X_{0}\right] \\
& =S(t)\left(X_{0}-\mathbb{E}\left[X_{0}\right]\right)+\int_{0}^{t} S(t-s) \mathrm{d} W(s),
\end{aligned}
$$

i.e., if $X$ is a mild solution of (3.4) with initial condition $X_{0}$, then $X-\mathbb{E}[X]$ is a mild solution of (3.4) with initial condition $X_{0}-\mathbb{E}\left[X_{0}\right]$. The assertion follows by an application of Theorem 4.2 to the transformed equation.

Acknowledgements The work of Annika Lang and Christoph Schwab was supported in part by ERC AdG No. 247277. Stig Larsson was supported by the Swedish Research Council VR. Part of this work was performed at the Mathematical Research Institute (FIM) of ETH Zürich during a visit of Stig Larsson to ETH.

\section{References}

1. Chegini, N., Stevenson, R.: Adaptive wavelet schemes for parabolic problems: Sparse matrices and numerical results. SIAM J. Numer. Anal. 49(1), 182-212 (2011)

2. Chow, P.L.: Stochastic Partial Differential Equations, Applied Mathematics and Nonlinear Science Series. Chapman \& Hall/CRC, Boca Raton, FL (2007)

3. Da Prato, G., Zabczyk, J.: Stochastic Equations in Infinite Dimensions, Encyclopedia of Mathematics and Its Applications, vol. 44. Cambridge University Press, Cambridge, MA (1992)

4. Ikeda, N., Watanabe, S.: Stochastic Differential Equations and Diffusion Processes, North-Holland Mathematical Library, vol. 24, 2nd edn. North-Holland, Amsterdam; Tokyo, Kodansha Ltd. (1989)

5. Peszat, S., Zabczyk, J.: Stochastic Partial Differential Equations with Lévy Noise, Encyclopedia of Mathematics and its Applications, vol. 113. Cambridge University Press, Cambridge, MA (2007)

6. Prévôt, C., Röckner, M.: A Concise Course on Stochastic Partial Differential Equations, Lecture Notes in Mathematics, vol. 1905. Springer, Berlin (2007)

7. Schwab, Ch., Stevenson, R.: Adaptive wavelet algorithms for elliptic PDE's on product domains. Math. Comput. 77(261), 71-92 (2008)

8. Schwab, Ch., Stevenson, R.: Space-time adaptive wavelet methods for parabolic evolution problems. Math. Comput. 78(267), 1293-1318 (2009)

9. Schwab, Ch., Süli, E.: Adaptive Galerkin approximation algorithms for Kolmogorov equations in infinite dimensions. Stoch. PDE: Anal. Comp. 1(1), 204-239 (2013)

10. Schwab, Ch., Todor, R.A.: Sparse finite elements for elliptic problems with stochastic loading. Numer. Math. 95(4), 707-734 (2003) 